\title{
Stromal p16 Overexpression in Gastric-type Mucinous Carcinoma of the Uterine Cervix
}

\author{
TAEK CHUNG ${ }^{1 *}$, SUNG-IM DO $^{2 *}$, KIYONG NA $^{1}$, GEON KIM $^{3}$, YOUNG IN JEONG $^{3}$, \\ YOUN WHA KIM ${ }^{3}$ and HYUN-SOO KIM ${ }^{1}$ \\ ${ }^{1}$ Department of Pathology, Severance Hospital, Yonsei University College of Medicine, Seoul, Republic of Korea; \\ ${ }^{2}$ Department of Pathology, Kangbuk Samsung Hospital, \\ Sungkyunkwan University School of Medicine, Seoul, Republic of Korea; \\ ${ }^{3}$ Department of Medicine, Graduate School, Kyung Hee University, Seoul, Republic of Korea
}

\begin{abstract}
Background/Aim: Gastric-type mucinous carcinoma $(M C-G)$ of the uterine cervix displays distinct morphological features and an aggressive clinical course. The expression status of p16 in the stroma has not been investigated in adenocarcinoma of the uterine cervix. Stromal p16 expression was evaluated in endocervical adenocarcinomas, including usual-type endocervical adenocarcinoma (UEA), intestinal-type mucinous carcinoma (MC-I), and MC-G. Whether stromal p16 expression varied significantly according to the histological subtype and whether the expression status is associated with clinicopathological characteristics of $M C-G$ was also investigated. Materials and Methods: Immunostaining of p16 was performed for 24, 19, and 18 cases of UEA, MC-I, and $M C-G$, respectively. Results: UEA and MC-I subtypes exhibited horizontally continuous, strong nuclear p16 immunoreactivity in the tumor cells, whereas none of the MC-G cases showed diffuse and strong nuclear immunoreactivity for p16 in the tumor cells. Instead, 10 (55.6\%) cases of MC-G displayed moderately to strongly positive p16 expression in the stroma. Stromal p16 expression of $M C-G$ was significantly higher than that of normal cervix, UEA, and MC-I. Metastatic MC-G had significantly higher stromal pl6 expression than primary $M C-G$. Further, stromal p16 overexpression in $M C-G$ was associated with advanced stage, parametrial invasion, and lymphovascular invasion. Conclusion: Stromal p16 expression
\end{abstract}

This article is freely accessible online.

*These Authors contributed equally to this study.

Correspondence to: Hyun-Soo Kim, Department of Pathology, Severance Hospital, Yonsei University College of Medicine, 50-1, Yonsei-ro, Seodaemun-gu, Seoul 03722, Republic of Korea. Tel: +82 222281794, +82 23620860, e-mail: hyunsookim@yuhs.ac

Key Words: Uterus, cervix, gastric-type mucinous carcinoma, stroma, p16, immunohistochemistry. of $M C-G$ was significantly higher than that of normal cervix and other histological subtypes of adenocarcinoma and was associated with advanced stage, parametrial invasion, and lymphovascular invasion, reflecting the aggressive behavior of $M C-G$. Our observations suggest that stromal p16 expression is involved in the development and progression of $M C-G$.

The number of cases of adenocarcinoma of the uterine cervix observed in routine histopathological practice has increased substantially from those reported several decades ago (1). Endocervical adenocarcinoma represents approximately $20 \%$ to $25 \%$ of cervical carcinomas $(2,3)$. It can be classified into two main types of tumors, those related to high-risk human papillomavirus (HPV) and those unrelated to high-risk HPV (4). The former, which represent the vast majority of endocervical adenocarcinoma, are referred to as endocervical adenocarcinomas of usual type (UEA) and mucinous carcinomas of intestinal type (MC-I), while the latter are dominated by mucinous carcinomas of gastric type (MC-G) (5-7). MC-G of the uterine cervix is a newly-recognized variant of endocervical adenocarcinoma, with distinctive morphological features (7). In contrast to UEA or MC-I, MC-G is frequently located in the upper endocervix and presents as a bulky cervix, without a welldemarcated mass because of its highly infiltrating pattern of growth (8). MC-G is associated with aggressive behavior, and its clinical outcome is worse than that of $\operatorname{UEA}(2,9,10)$.

In recent years, immunostaining of p16 has been used as a diagnostic aid in gynecological pathology (11). Horizontally continuous and moderate-to-strong nuclear immunoreactivity (block positivity) for p16 reflects an association with highrisk HPV infection and is used as a confirmatory tool for the diagnosis of HPV-related tumors, including squamous cell carcinoma, UEA, and MC-I $(12,13)$. In contrast, MC-G unrelated to high-risk HPV displays variable patchy p16 immunoreactivity ranging from negative to focal and weak expression with scattered faint foci and interspersed individual negative cells (4). However, unlike p16 expression, 
MC-G exhibit either strong diffuse overexpression of $\mathrm{p} 53$ (missense mutation pattern) or are completely negative for p53 expression (nonsense/frameshift mutation pattern), with both patterns being highly correlated with a TP53 mutation (14). Moreover, both UEA and MC-I exhibit patchy and weak p53 expression (wild-type pattern). Taken together, these findings demonstrate that immunohistochemical analysis of p16 and p53 expression is useful for distinguishing endocervical adenocarcinoma subtypes.

Recently, during the diagnosis of endocervical adenocarcinomas in routine practice, we observed p16 expression in the stromal cells, as well as in the neoplastic glandular epithelial cells. Stromal p16 levels seemed to vary among cases, depending particularly on the histological subtype. Although p16 is commonly used as a biomarker for diagnosing gynecological malignancies, its expression in the stromal component of endocervical adenocarcinomas has not been reported to our knowledge. In this study, we evaluated stromal p16 immunoreactivity in malignant glandular lesions of the uterine cervix and investigated whether stromal p16 expression varied significantly according to the histological subtype. In addition, we examined whether the status of stromal p16 expression was associated with the clinicopathological characteristics of MC-G.

\section{Materials and Methods}

Patient selection and tissue specimens. The study protocol (4-20180150) was reviewed and approved by the Institutional Review Board of the Severance Hospital (Seoul, Republic of Korea). Between March 2016 and August 2017, tissue samples of UEA, MC-I, and MC-G, were obtained from 24, 19, and 18 patients, respectively. Representative photomicrographs displaying characteristic histopathological features and immunophenotype of each subtype are presented in Figure 1. Corresponding tissue samples from metastatic MC-G were obtained from 11 of the 18 patients with MC-G. Normal cervical tissue samples were also obtained from 10 patients who underwent total hysterectomy for uterine leiomyomata. No patient underwent preoperative neoadjuvant chemotherapy or neoadjuvant concurrent chemoradiation therapy. Clinicopathological information was obtained from electronic medical records and pathology reports. The clinical and pathological details that were reviewed included age of patient at initial diagnosis, greatest dimension of tumor, parametrial extension, International Federation of Gynecology and Obstetrics (FIGO) stage (15), and lymphovascular invasion. Pathological diagnoses were established following the criteria of the World Health Organization Classification of Tumours of Female Reproductive Organs, revised in 2014 (7).

Pathological examination. Resected tissues obtained by gynecological oncology surgeons were initially examined by pathologists prior to fixation in $10 \%$ neutral-buffered formalin for 12-24 hours. The fixed tissues were then thoroughly examined macroscopically and sectioned. After processing with an automatic tissue processor (Peloris II; Leica Microsystems, Newcastle Upon Tyne, UK), the sections were embedded in paraffin blocks. Four micrometer-thick slices were serially cut from each formalin-fixed, paraffin-embedded tissue block using a rotary microtome (RM2245; Leica Microsystems), and mounted onto microscope slides. The slices were stained with hematoxylin and eosin using an automatic staining instrument (Ventana Symphony System; Ventana Medical Systems, Tucson, AZ, USA). After staining, a glass coverslip was applied to the slides, which were then submitted to a Board-certified pathologist specializing in gynecological oncology. The pathologist examined the slides by light microscopy (BX43 System Microscope; Olympus, Tokyo, Japan) and issued definitive pathological diagnoses.

Immunohistochemistry. The 4- $\mu$ m-thick, formalin-fixed, paraffinembedded slices were deparaffinized and rehydrated with xylene and alcohol solutions. Immunostaining was performed using an automatic immunostaining instrument (Ventana Benchmark XT; Ventana Medical Systems) according to the manufacturer's recommendations $(14,16-26)$. Antigen retrieval was performed using Cell Conditioning Solution CC1 (Ventana Medical Systems). The slices were incubated with primary antibodies against p16 (prediluted, clone E6H4; Ventana Medical Systems) and p53 (1:300, clone DO-7; Novocastra, Leica Biosystems, Newcastle Upon Tyne, UK). After chromogenic visualization using an ultraView Universal DAB Detection Kit (Ventana Medical Systems), slices were counterstained with hematoxylin and sealed with coverslips. Appropriate positive and negative controls were concurrently stained to validate the staining methods. Negative controls were prepared by substituting non-immune serum for the primary antibody so that no detectable staining was obvious. The p16 immunostaining pattern was interpreted as block positivity when p16 expression was strong and horizontally continuous, and included nuclear or nuclear plus cytoplasmic staining. All other p16 immunostaining patterns, including focal or wispy nuclear staining, and blob-like, puddled, or scattered cytoplasmic staining were interpreted as patchy positivity $(16,26,27)$. The p53 immunostaining pattern was defined as a missense mutation pattern, nonsense/frameshift mutation pattern, or wild-type pattern when the p53 expression was diffuse and strong (more than $60 \%$ of tumor cell nuclei), absent (fewer than $5 \%$ ), or focal and weakly positive, respectively $(7,14)$.

Interpretation of stromal p16 immunoreactivity. The percentage of p16-positive stromal cells and staining intensity were assessed as previously described (28-30). The estimated percentages were categorized as follows: absent: score 0 , less than $10 \%$ : score $1,10-$ $24 \%$ : score $2,25-49 \%$ : score 3 , and $50 \%$ or more: score 4 . The staining intensity was graded as follows: negative: score 0 , weak: score 1, moderate: score 2, and strong: score 3. The subcellular locations of the p16-positive signals (nuclear or cytoplasmic) were also estimated. The final immunoreactivity score was calculated as the product of the percentage and staining intensity scores, resulting in scores of $0,1,2,3,4,6,8,9$, or 12 . The immunoreactivity scores were categorized as follows: negative: score; weakly positive: scores 1, 2, and 3; moderately positive: scores 4 and 6; strongly positive: scores 8,9 , and 12 . In addition, the stromal p16 expression was dichotomized into high (moderately-to-strongly positive) and low (weakly positive/negative) expression for statistical analysis.

Statistical analysis. A linear-by-linear test was performed to examine differences in p16 immunoreactivity according to the histological subtype and to compare the stromal p16 expression between primary 


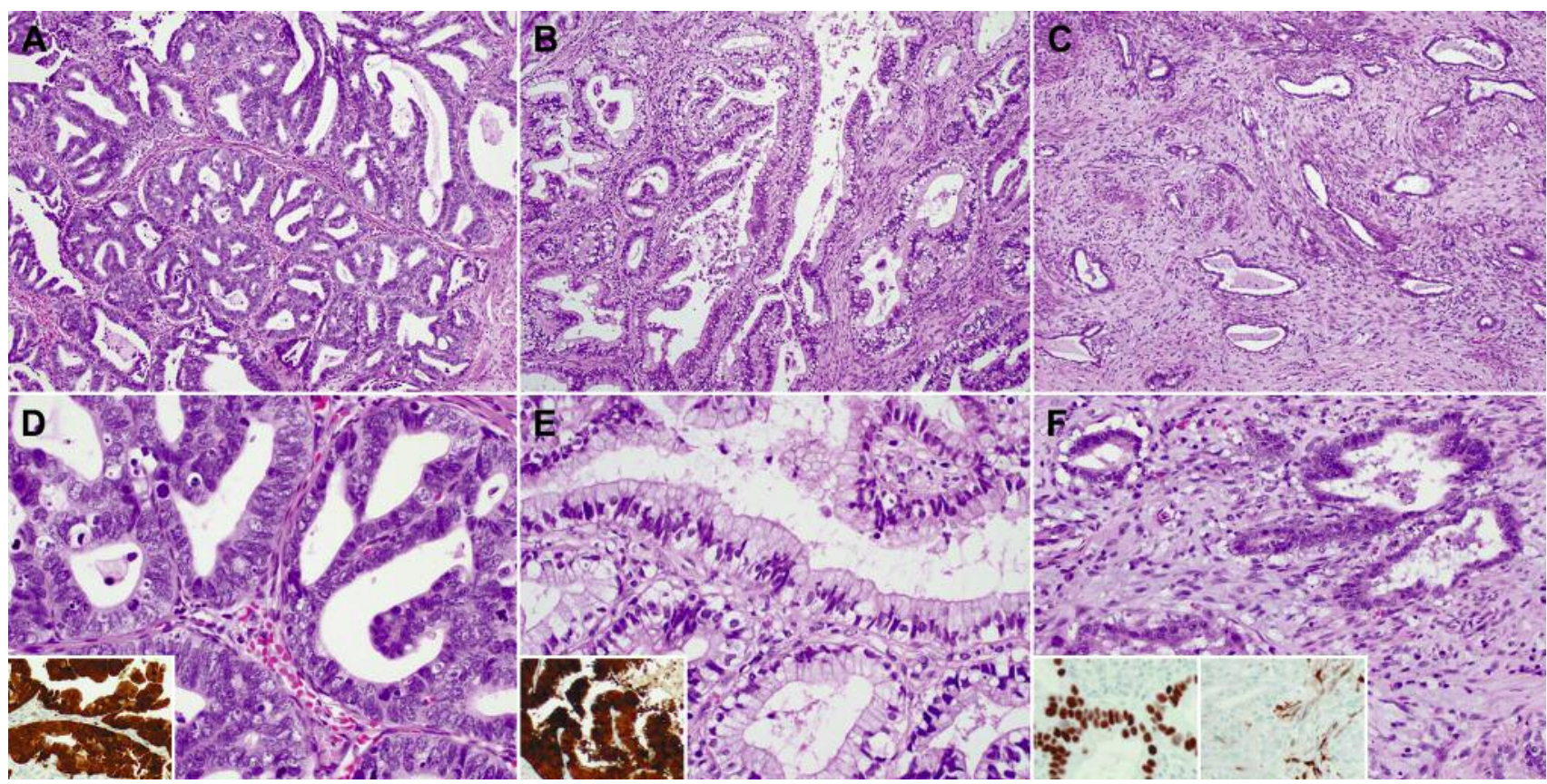

Figure 1. Representative photomicrographs showing characteristic histopathological features of endocervical adenocarcinoma. A: Usual-type endocervical adenocarcinoma (UEA) displays a glandular growth pattern with occasional cribriform architecture and papillary projections into glandular lumina. B: Intestinal-type mucinous carcinoma (MC-I) shows a similar pattern of growth to that of UEA, but the glandular epithelium consists of mucin-containing columnar cells. C: Gastric-type mucinous carcinoma (MC-G) shows irregularly dilated, simple glands showing a haphazard growth in desmoplastic stroma. D: High-power view of UEA. The tumor cells are columnar without mucin, with high nuclear-tocytoplasmic ratio. Inset: diffuse and strong expression (block positivity) for p16 in tumor cells. E: High-power view of MC-I. Columnar cells with pale, mucin-containing cytoplasm and basally located nuclei are noted. Inset: block positivity for p16 in tumor cells. F: High-power view of MC$G$. The tumor cells possess ample, eosinophilic, or clear cytoplasm. Their nuclei have mild-to-moderate atypia. Inset: Left: diffuse, strong expression for p53 (TP53 missense mutation pattern); right: negative p16 immunoreactivity in tumor cells. Original magnifications: A-C, 100x; D-F, 400x.

and metastatic MC-G. A Fisher's exact test was performed to determine whether there was a significant association between the stromal p16 expression status and the clinicopathological characteristics of MC-G. Statistical analyses were conducted using SPSS version 18.0 (SPSS, Inc., Chicago, IL, USA). Differences with $p$-values less than 0.05 were considered significant.

HPV genotype assay. Polymerase chain reaction (PCR)-based microarray analysis for HPV genotyping was performed using a commercially available HPV 9G DNA chip (BMT HPV 9G DNA Chip; Biometrix Technology, Chuncheon, Republic of Korea) (31, 32 ). The $9 \mathrm{G}$ microarray detected the presence of 14 high-risk (types $16,18,31,33,35,39,45,51,52,56,58,59,66$, and 68) and five low-risk (types $6,11,34,40$, and 42) HPV types; the analyses were performed according to the manufacturer's recommendations (3336). Briefly, the PCR mixture consisted of $10 \mu \mathrm{l}$ of the extracted target DNA, $10 \mu$ of BMT Primer set (Biometrix Technology), and PCR premix (Biometrix Technology) that contained dNTP and Taq DNA polymerase in an amplification buffer. Amplification was performed using the following protocol: predenaturation for $5 \mathrm{~min}$ at $94^{\circ} \mathrm{C} ; 40$ sequential cycles of denaturation at $94^{\circ} \mathrm{C}$ for $30 \mathrm{~s}$; annealing at $45^{\circ} \mathrm{C}$ for $30 \mathrm{~s}$; elongation at $72^{\circ} \mathrm{C}$ for $30 \mathrm{~s}$; followed by a final 5-min elongation step at $72^{\circ} \mathrm{C}$. The PCR products were electrophoresed in $2 \%$ agarose gels to confirm amplification. Each hybridization chamber of the $9 \mathrm{G}$ chip was covered with a mixture of the hybridization solution $(35 \mu \mathrm{l})$ and the polymerase chain reaction product $(15 \mu \mathrm{l})$ and incubated at $23-26^{\circ} \mathrm{C}$ for $30 \mathrm{~min}$. After washing, array images were scanned and imaged using a fluorescent scanner (ScanArray GX Microarray Scanner; PerkinElmer Life and Analytical Sciences, Waltham, MA, USA).

\section{Results}

Baseline characteristics. The age of patients with MC-G ranged from 39 to 70 years. Nine $(50.0 \%)$ patients were 56 years or older. All patients underwent radical hysterectomy with bilateral salpingo-oophorectomies, bilateral pelvic lymph node dissections, with/without para-aortic lymph node dissection or sampling. The greatest dimension of tumor ranged from 2.8 to $6.7 \mathrm{~cm}$. Twelve $(66.7 \%)$ tumors were greater than $4.0 \mathrm{~cm}$. Parametrial extension was identified in $10(55.6 \%)$ patients. The FIGO stage distribution was as follows: IIA, $27.8 \%$ (5/18); IIB, 33.3\% (6/18); and III, 38.9\% (7/18). The metastatic sites included pelvic lymph nodes in 
Table I. p16 Expression in tumor cells according to histological subtype of endocervical adenocarcinoma.

\begin{tabular}{|c|c|c|c|c|c|c|c|}
\hline \multirow[t]{2}{*}{ Subtype } & \multirow[b]{2}{*}{ Type } & \multirow[b]{2}{*}{ Total, $\mathrm{n}$} & \multicolumn{4}{|c|}{ p16 expression in tumor cells, $\mathrm{n}(\%)$} & \multirow[b]{2}{*}{$p$-Value } \\
\hline & & & Negative & Weakly positive & Moderately positive & Strongly positive & \\
\hline \multirow[t]{2}{*}{ Mucinous carcinoma } & Gastric & 18 & $15(83.3)$ & $3(16.7)$ & $0(0.0)$ & $0(0.0)$ & \\
\hline & Intestinal & 19 & $0(0.0)$ & $0(0.0)$ & $0(0.0)$ & $19(100.0)$ & $<0.001^{\mathrm{a}}$ \\
\hline Endocervical adenocarcinoma & Usual & 24 & $0(0.0)$ & $0(0.0)$ & $0(0.0)$ & $24(100.0)$ & $<0.001^{\mathrm{a}}$ \\
\hline
\end{tabular}

${ }^{a}$ Versus mucinous carcinoma, gastric type.

Table II. Stromal p16 expression according to histological subtype of endocervical adenocarcinoma.

\begin{tabular}{|c|c|c|c|c|c|c|c|}
\hline \multirow[t]{2}{*}{ Subtype } & \multirow[b]{2}{*}{ Type } & \multirow[b]{2}{*}{ Total, $\mathrm{n}$} & \multicolumn{4}{|c|}{ Stromal p16 expression, n (\%) } & \multirow[b]{2}{*}{$p$-Value } \\
\hline & & & Negative & Weakly positive & Moderately positive & Strongly positive & \\
\hline \multirow[t]{2}{*}{ Mucinous carcinoma } & Gastric & 18 & $2(11.1)$ & $6(33.3)$ & $7(38.9)$ & $3(16.7)$ & \\
\hline & Intestinal & 19 & $12(63.2)$ & $5(26.3)$ & $2(10.5)$ & $0(0.0)$ & $<0.001^{\mathrm{a}}$ \\
\hline Endocervical adenocarcinoma & Usual & 24 & $14(58.3)$ & $7(29.2)$ & $3(12.5)$ & $0(0.0)$ & $\begin{array}{r}<0.001^{\mathrm{a}} \\
0.753^{\mathrm{b}}\end{array}$ \\
\hline
\end{tabular}

aVersus mucinous carcinoma, gastric type; ${ }^{\mathrm{b}}$ versus mucinous carcinoma, intestinal type.

Table III. Stromal p16 expression among samples of normal cervix and primary and metastatic gastric-type mucinous carcinoma.

\begin{tabular}{lllllll}
\hline Subtype & & \multicolumn{3}{c}{ Stromal p16 expression, $\mathrm{n}(\%)$} \\
\cline { 3 - 5 } & Total, $\mathrm{n}$ & Negative & Weakly positive & Moderately positive & Strongly positive & $p$-Value \\
\hline Normal cervix & 10 & $7(70.0)$ & $3(30.0)$ & $0(0.0)$ & $0(0.0)$ \\
Primary mucinous carcinoma, gastric type & 19 & $2(11.1)$ & $6(33.3)$ & $7(38.9)$ & $3(16.7)$ & $<0.001^{\mathrm{a}}$ \\
Metastatic mucinous carcinoma, gastric type & 11 & $0(0.0)$ & $2(18.2)$ & $3(27.3)$ & $6(54.5)$ & $<0.001^{\mathrm{a}}$ \\
& & & & & $0.036^{\mathrm{b}}$ \\
\hline
\end{tabular}

${ }^{a}$ Versus normal cervix; ${ }^{\mathrm{b}}$ versus primary mucinous carcinoma, gastric type.

$5 / 11(45.5 \%)$, ovary in $3 / 11(27.3 \%)$, pelvic peritoneum in $2 / 11(18.2 \%)$, and abdominal peritoneum in $1 / 11(9.1 \%)$. Lymphovascular invasion was observed in nine $(50.0 \%)$ cases. High-risk HPV was detected in all cases of UEA (24/24) and MC-I (19/19). The most common HPV genotype found was type $18(24 / 43,55.8 \%)$. Type 16 , detected in $30.2 \%(13 / 43)$ of the cases, was the second most common high-risk HPV genotype in this study. In contrast, none of the MC-G cases were found to be infected with HPV.

Expression of p16 and p53 in tumor cells. Consistent with the HPV results, all cases of UEA (24/24) and MC-I (19/19) demonstrated p16 block positivity in the nuclei and cytoplasm of tumor cells, whereas in the majority of MC-G cases $(15 / 18,83.3 \%)$ p16 expression was absent from the tumor cells (Table I). The remaining three cases of MC-G $(16.7 \%)$ exhibited patchy and weak p16 immunoreactivity in the cytoplasm of tumor cells.

The p53 immunostaining patterns of MC-G corresponded with either the missense mutation pattern $(13 / 18,72.2 \%)$ or the nonsense/frameshift mutation pattern $(5 / 18,27.8 \%)$. All cases of UEA (24/24) and MC-I (19/19) showed the wildtype pattern of p53 expression.

Expression of pl6 in stromal cells. Representative photomicrographs showing stromal p16 expression in MC-G 

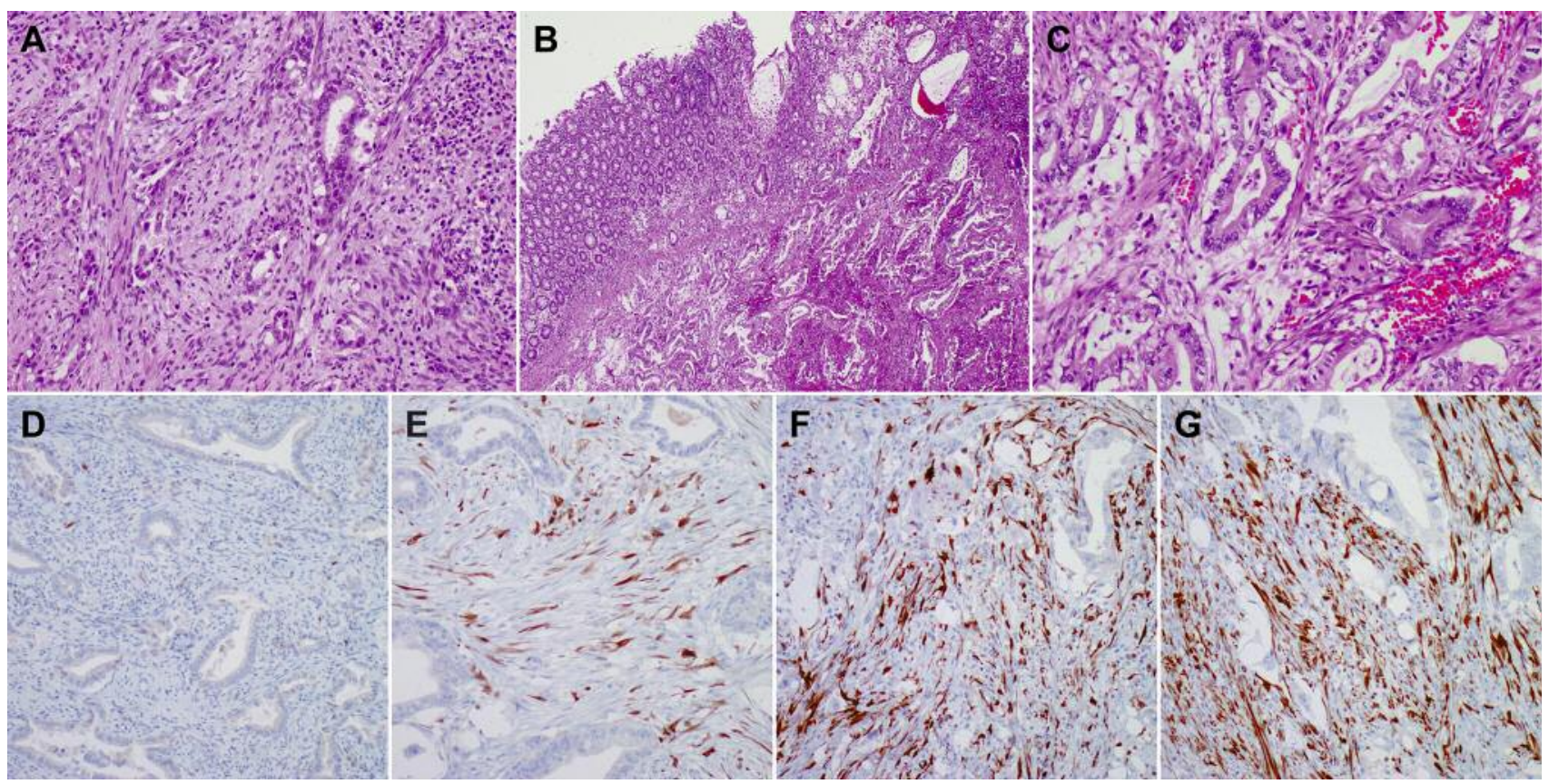

Figure 2. Representative photomicrographs showing stromal p16 expression in gastric-type mucinous carcinoma (MC-G). A: Primary MC-G. Variable-sized, irregularly spaced tumor glands invade the desmoplastic stroma. Inflammatory cell infiltrates are associated. B: Metastatic MC-G. The tumor tissue extends through the proper muscle and submucosa into the mucosa of the rectum. C: High-power view of metastatic MC-G. D: Negative, E: moderately positive, and F: strongly positive stromal p16 expression in primary MC-G. F: Strongly positive stromal p16 expression in metastatic MC-G. Original magnifications: A, 200x; B, 40x; C-G, 200x.

are presented in Figure 2. Of the 18 cases of MC-G, $10(55.6 \%)$ showed moderately to strongly positive p16 expression in the nuclei and cytoplasm of stromal cells, whereas six (33.3\%) and two $(11.1 \%)$ cases demonstrated weakly positive and negative expression, respectively. No cases of UEA or MC-I exhibited strongly positive stromal p16 expression. Only two (10.5\%) cases of MC-I and three (12.5\%) cases of UEA showed moderately positive p16 expression in the stromal cells. Most cases of MC-I $(12 / 19,63.2 \%)$ did not show stromal p16 expression. Similarly, in more than half of UEA cases (14/24, $58.3 \%$ ) stromal p16 immunoreactivity was absent. As shown in Table II, stromal p16 levels were significantly higher in MC-G compared with those in UEA $(p<0.001)$ and MC-I $(p<0.001)$. No significant difference in stromal p16 expression was observed between MC-I and UEA ( $p=0.753)$.

The stromal p16 levels in normal cervix, primary MC-G, and metastatic MC-G tissue samples are presented in Table III. Weakly positive cytoplasmic p16 immunoreactivity was observed in seven $(70.0 \%)$ out of the 10 normal cervical tissue samples, while in the remaining three $(30.0 \%)$ samples, stromal p16 expression was not detected. The difference in stromal p16 expression between primary MC-G and normal cervix was statistically significant $(p<0.001)$. Moreover, stromal p16 expression in metastatic $\mathrm{MC}-\mathrm{G}$ was significantly higher than that in the primary MC$\mathrm{G}$ tissue samples $(p=0.036)$. More than half $(6 / 11,54.5 \%)$ of the metastatic MC-G cases exhibited strongly positive p16 immunoreactivity in the stromal cells.

Association between stromal p16 expression and clinicopathological characteristics of $M C-G$. Relationships between the stromal p16 expression status and clinicopathological characteristics of MC-G are summarized in Table IV. Significant positive correlations were observed between stromal p16 overexpression and both advanced stage $(p=0.007)$ and lymphovascular invasion $(p=0.015)$. There was a marginally significant association between high stromal p16 expression and parametrial extension $(p=0.054)$. However, the associations of stromal p16 expression with patient age and the greatest dimension of tumor were not statistically significant.

\section{Discussion}

Unlike studies on p16 expression in epithelial lesions, there have been very few studies applying p16 immunostaining to 
the stromal component of gynecological lesions. In this study, we investigated stromal p16 expression in adenocarcinomas of the uterine cervix. Firstly, we observed a significant increase in stromal p16 expression in primary MC-G tissues compared with that in normal cervical tissues. More than half of the MC-G cases showed moderately-tostrongly positive p16 immunoreactivity in the stromal cells, whereas none of the normal cervical tissue samples exhibited this level of positive stromal p16 expression, suggesting that p16 overexpression in the stroma might modulate the tumor microenvironment to promote $\mathrm{MC}-\mathrm{G}$ development. Secondly, we noted that metastatic MC-G exhibited significantly higher p16 levels in the stromal cells compared with primary MC$\mathrm{G}$. The proportion of metastatic MC-G cases with strongly positive stromal p16 immunoreactivity (54.5\%) was significantly higher than that of primary MC-G $(16.7 \%)$, suggesting that stromal p16 expression may influence the metastatic progression of MC-G. Our data are consistent with previous reports that p16 promotes invasiveness through interactions with other molecules associated with tumor cell migration and invasion $(28,37-40)$, as well as with our recent studies demonstrating stromal p16 overexpression in ovarian and endometrial carcinomas (29, 30). We have also shown here that ovarian carcinoma displays significantly higher stromal p16 expression than benign and borderline ovarian tumors. Similarly, significantly higher stromal p16 expression was observed in endometrial carcinomas than in benign and precancerous lesions of the endometrium. Another study on stromal p16 expression in breast carcinoma reported that ductal carcinoma in situ with increased stromal p16 expression tended to show estrogen receptor negativity and a high $\mathrm{Ki}$ 67 labeling index, and that stromal p16 overexpression is an independent predictor of recurrence with a higher relative risk than other established prognostic parameters (41).

The overexpression of $\mathrm{p} 16$ occurs in malignant tumors via two different mechanisms. The first is an abnormality in the retinoblastoma protein pathway. Physiologically, p16 inactivates cyclin-dependent kinase 4/6, blocking phosphorylation of the retinoblastoma protein and induces cell-cycle arrest. Loss of p16 function allows deregulated cellular proliferation (42). Paradoxically, some malignant tumors exhibit p16 overexpression, HPV-related tumors being a well-known example. The HPV oncoprotein E7 inactivates the retinoblastoma protein, which results in the release of p16 due to its negative feedback control and up-regulation of CDKN2A/p16 (43). Given the fact that MC-G is unrelated to HPV infection, our observation of stromal p16 overexpression in MC-G may be explained by a positive feedback mechanism caused by deregulation of the retinoblastoma protein pathway (43). The second mechanism of p16 overexpression is oncogene-induced cell senescence. Based on the notion that p16 induces cellular senescence (28), we also postulated that
Table IV. Association between stromal p16 expression and clinicopathological characteristics of patients with cervical gastric-type mucinous carcinoma.

\begin{tabular}{|c|c|c|c|c|}
\hline \multirow[t]{2}{*}{ Characteristic } & \multirow[b]{2}{*}{$\begin{array}{c}\text { Total, } \\
\mathrm{n}\end{array}$} & \multicolumn{2}{|c|}{$\begin{array}{c}\text { Stromal p16 expression, } \\
\mathrm{n}(\%)\end{array}$} & \multirow[t]{2}{*}{$p$-Value } \\
\hline & & $\begin{array}{c}\text { Moderately/ } \\
\text { strongly } \\
\text { postive }\end{array}$ & $\begin{array}{l}\text { Negative/ } \\
\text { weakly } \\
\text { positive }\end{array}$ & \\
\hline \multicolumn{5}{|l|}{ Age (years) } \\
\hline$>55$ & 9 & $6(66.7)$ & $3(33.3)$ & 0.637 \\
\hline$\leq 55$ & 9 & $4(44.4)$ & $5(55.6)$ & \\
\hline \multicolumn{5}{|c|}{ Greatest dimension $(\mathrm{cm})$} \\
\hline$>4.0$ & 12 & $7(58.3)$ & $5(41.7)$ & 1.000 \\
\hline$\leq 4.0$ & 6 & $3(50.0)$ & $3(50.0)$ & \\
\hline \multicolumn{5}{|c|}{ Parametrial extension } \\
\hline Present & 10 & $8(80.0)$ & $2(20.0)$ & 0.054 \\
\hline Absent & 8 & $2(25.0)$ & $6(75.0)$ & \\
\hline \multicolumn{5}{|l|}{ Stage } \\
\hline IIB-III & 13 & $10(76.9)$ & $3(23.1)$ & 0.007 \\
\hline IIA & 5 & $0(0.0)$ & $5(100.0)$ & \\
\hline \multicolumn{5}{|c|}{ Lymphovascular invasion } \\
\hline Present & 9 & $8(88.9)$ & $1(11.1)$ & 0.015 \\
\hline Absent & 9 & $2(22.2)$ & $7(77.8)$ & \\
\hline
\end{tabular}

stromal p16 overexpression is indicative of stromal cell senescence and that the senescent stroma contributes to disease progression by secreting inflammatory mediators, cytokines, and proteases, which together mediate tumor progression. Further studies are necessary to clarify the mechanism of stromal $\mathrm{p} 16$ expression in MC-G.

We further observed a significant increase in stromal p16 expression in MC-G compared with that in UEA and MC-I. UEA and MC-I showed moderate p16 positivity in $12.5 \%$ and $10.5 \%$ of the cases examined, respectively. In contrast, $55.6 \%$ of the MC-G cases had moderately-to-strongly positive $\mathrm{p} 16$ expression in the stromal cells. Consistent with this finding, we have previously observed differences in stromal p16 expression according to the histological subtype in ovarian and endometrial carcinomas (29, 30). Among endometrial carcinomas, serous tumors show significantly higher p16 expression than endometrioid tumors. Similarly, serous endometrial intraepithelial carcinoma, a precancerous lesion of serous carcinoma, shows higher stromal p16 expression than atypical hyperplasia/endometrioid intraepithelial neoplasia, a precancerous lesion of endometrioid carcinoma. These data suggest that stromal p16 expression may, at least partly, be reflective of the more aggressive behavior and worse prognosis of endometrial serous carcinoma than those of endometrial endometrioid carcinoma. Our observation that MC-G displays higher stromal p16 expression than UEA and $\mathrm{MC}-\mathrm{I}$ may also reflect the more aggressive nature of $\mathrm{MC}-\mathrm{G}$ 
compared with that of the other two histological subtypes of endocervical adenocarcinoma. This concept is further supported by our observations that stromal p16 overexpression is associated with advanced stage, parametrial extension, and lymphovascular invasion in patients with MCG. Further studies on the clinicopathological and prognostic significance of stromal p16 expression of MC-G, with a larger patient cohort are necessary.

In conclusion, we demonstrated stromal p16 expression in tissue samples of endocervical adenocarcinoma using immunohistochemistry. Stromal p16 expression in metastatic MC-G was significantly higher than that in primary MC-G, which in turn was significantly higher than that in normal cervical tissue samples. Moreover, the stromal p16 expression in $M C-G$ was significantly higher than that in UEA and MC-I. In patients with MC-G, stromal p16 overexpression was associated with advanced stage, parametrial extension, and lymphovascular invasion. Collectively, our data suggest that p16 overexpression in stromal cells is involved in the development and progression of $\mathrm{MC}-\mathrm{G}$, as well as in its aggressive behavior.

\section{Acknowledgements}

This research was supported by the Basic Science Research Program through the National Research Foundation of Korea (NRF) funded by the Ministry of Education (2016R1D1A1B03935584) and a Korean Society of Pathology Grant (2016).

\section{References}

1 Young RH and Clement PB: Endocervical adenocarcinoma and its variants: their morphology and differential diagnosis. Histopathology 41: 185-207, 2002.

2 Karamurzin YS, Kiyokawa T, Parkash V, Jotwani AR, Patel P, Pike MC, Soslow RA and Park KJ: Gastric-type endocervical adenocarcinoma: An aggressive tumor with unusual metastatic patterns and poor prognosis. Am J Surg Pathol 39: 1449-1457, 2015.

3 Smith HO, Tiffany MF, Qualls CR and Key CR: The rising incidence of adenocarcinoma relative to squamous cell carcinoma of the uterine cervix in the United States-A 24-year population-based study. Gynecol Oncol 78: 97-105, 2000.

4 Ronnett BM: Endocervical adenocarcinoma: selected diagnostic challenges. Mod Pathol 29(Suppl 1): S12-28, 2016.

5 McCluggage WG: New developments in endocervical glandular lesions. Histopathology 62: 138-160, 2013.

6 Mikami Y and McCluggage WG: Endocervical glandular lesions exhibiting gastric differentiation: an emerging spectrum of benign, premalignant, and malignant lesions. Adv Anat Pathol 20: 227-237, 2013.

7 Kurman RJ, Carcangiu ML, Herrington CS and Young RH: WHO Classification of Tumours of Female Reproductive Organs. Lyon, France: IARC, 2014.

8 Kojima A, Shimada M, Mikami Y, Nagao S, Takeshima N, Sugiyama T, Teramoto N, Kiyokawa T, Kigawa J, Nishimura R and Sankai Gynecology Study G: Chemoresistance of gastric- type mucinous carcinoma of the uterine cervix: A study of the Sankai Gynecology Study Group. Int J Gynecol Cancer 28: 99106, 2018.

9 Park KJ, Kiyokawa T, Soslow RA, Lamb CA, Oliva E, Zivanovic O, Juretzka MM and Pirog EC: Unusual endocervical adenocarcinomas: an immunohistochemical analysis with molecular detection of human papillomavirus. Am J Surg Pathol 35: 633-646, 2011.

10 Kusanagi Y, Kojima A, Mikami Y, Kiyokawa T, Sudo T, Yamaguchi $\mathrm{S}$ and Nishimura R: Absence of high-risk human papillomavirus (HPV) detection in endocervical adenocarcinoma with gastric morphology and phenotype. Am J Pathol 177: 21692175,2010 .

11 O'Neill CJ and McCluggage WG: p16 expression in the female genital tract and its value in diagnosis. Adv Anat Pathol 13: 815, 2006.

12 Mulvany NJ, Allen DG and Wilson SM: Diagnostic utility of p16 ${ }^{\text {INK4AA }}$ a reappraisal of its use in cervical biopsies. Pathology 40: 335-344, 2008.

13 Romagosa C, Simonetti S, Lopez-Vicente L, Mazo A, Lleonart ME, Castellvi J and Ramon y Cajal S: p16(INK4A) overexpression in cancer: A tumor-suppressor gene associated with senescence and high-grade tumors. Oncogene 30: 2087-2097, 2011.

$14 \mathrm{Na} \mathrm{K}$, Sung JY and Kim HS: TP53 Mutation status of tuboovarian and peritoneal high-grade serous carcinoma with a wildtype p53 immunostaining pattern. Anticancer Res 37: 66976703, 2017.

15 Pecorelli S, Zigliani L and Odicino F: Revised FIGO staging for carcinoma of the cervix. Int J Gynaecol Obstet 105: 107-108, 2009.

$16 \mathrm{Na} \mathrm{K}$ and Kim HS: Clinicopathologic and molecular characteristics of mesonephric adenocarcinoma arising from the uterine body. Am J Surg Pathol, 2017. doi: 10.1097/PAS.00000 00000000991. [Epub ahead of print]

17 Do SI, Kim HS, Kim K, Lee H, Do IG, Kim DH, Chae SW and Sohn JH: Predictive and prognostic value of sphingosine kinase 1 expression in patients with invasive ductal carcinoma of the breast. Am J Transl Res 9: 5684-5695, 2017.

18 Do SI, Kim HS, Kim K, Lee H, Do IG, Kim DH, Chae SW and Sohn JH: Predictive value of sphingosine kinase 1 expression in papillary thyroid carcinoma. Anticancer Res 37: 5399-5405, 2017.

19 Jang MI, Sung JY, Kim JY and Kim HS: Clinicopathological characteristics of metaplastic papillary tumor of the fallopian tube. Anticancer Res 37: 3693-3701, 2017.

20 Kim JY, Na K and Kim HS: Clinicopathological characteristics of mitotically-active cellular fibroma of the ovary: A singleinstitutional experience. Anticancer Res 37: 2557-2564, 2017.

$21 \mathrm{Na} \mathrm{K}$, Kim EK, Jang W and Kim HS: CTNNB1 mutations in ovarian microcystic stromal tumors: identification of a novel deletion mutation and the use of pyrosequencing to identify reported point mutation. Anticancer Res 37: 3249-3258, 2017.

22 Na K, Park SY and Kim HS: Clinicopathological characteristics of primary ovarian adenomyoma: A single-institutional experience. Anticancer Res 37: 2565-2574, 2017.

23 Sung JY, Na K and Kim HS: Down-regulation of inositol polyphosphate 4-phosphatase type II expression in colorectal carcinoma. Anticancer Res 37: 5525-5531, 2017.

24 Kim JY, Do SI, Bae GE and Kim HS: B-Cell translocation gene 1 is down-regulated by promoter methylation in ovarian carcinoma. J Cancer 8: 2669-2675, 2017. 
25 Lee JY, Chung YS, Na K, Kim HM, Park CK, Nam EJ, Kim S, Kim SW, Kim YT and Kim HS: External validation of chemotherapy response score system for histopathological assessment of tumor regression after neoadjuvant chemotherapy in tubo-ovarian high-grade serous carcinoma. J Gynecol Oncol 28: e73, 2017.

$26 \mathrm{Na} \mathrm{K}$ and Kim HS: Clinicopathological characteristics of fallopian tube metastases from primary endometrial, cervical, and nongynecological malignancies: A single-institutional experience. Virchows Arch 471: 363-373, 2017.

27 Darragh TM, Colgan TJ, Thomas Cox J, Heller DS, Henry MR, Luff RD, McCalmont T, Nayar R, Palefsky JM, Stoler MH, Wilkinson EJ, Zaino RJ, Wilbur DC and Members of the LPWG: The Lower Anogenital Squamous Terminology Standardization project for HPV-associated lesions: background and consensus recommendations from the College of American Pathologists and the American Society for Colposcopy and Cervical Pathology. Int J Gynecol Pathol 32: 76-115, 2013.

$28 \mathrm{Na} \mathrm{K}$, Sung JY and Kim HS: Stromal p16 overexpression in adult granulosa cell tumors of the ovary. Anticancer Res 37: 2437-2444, 2017.

29 Yoon N, Yoon G, Park CK and Kim HS: Stromal p16 expression is significantly increased in malignant ovarian neoplasms. Oncotarget 7: 64665-64673, 2016.

30 Yoon G, Koh CW, Yoon N, Kim JY and Kim HS: Stromal p16 expression is significantly increased in endometrial carcinoma. Oncotarget 8: 4826-4836, 2017.

31 An H, Song KS, Nimse SB, Kim J, Nguyen VT, Ta VT, Sayyed DR and Kim T: HPV 9G DNA chip: 100\% clinical sensitivity and specificity. J Clin Microbiol 50: 562-568, 2012.

32 Jung HY, Han HS, Kim HB, Oh SY, Lee SJ and Kim WY: Comparison of analytical and clinical performance of HPV 9G DNA Chip, PANArray HPV Genotyping Chip, and HybridCapture II Assay in cervicovaginal swabs. J Pathol Transl Med 50: 138-146, 2016.

$33 \mathrm{Na} \mathrm{K}$, Sung JY and Kim HS: Clinicopathological characteristics of high-grade squamous intraepithelial lesions involving condyloma acuminatum. Anticancer Res 38: 1767-1774, 2018.

34 Yoon N, Kim JY and Kim HS: Clinical outcomes of advancedstage glassy cell carcinoma of the uterine cervix: A need for reappraisal. Oncotarget 7: 78448-78454, 2016.

35 Jung YY, Nahm JH and Kim HS: Cytomorphological characteristics of glassy cell carcinoma of the uterine cervix: Histopathological correlation and human papillomavirus genotyping. Oncotarget 7: 74152-74161, 2016.
36 Jun SY, Park ES, Kim J, Kang J, Lee JJ, Bae Y, Kim SI and Maeng LS: Comparison of the Cobas $4800 \mathrm{HPV}$ and HPV 9G DNA chip tests for detection of high-risk human papillomavirus in cervical specimens of women with consecutive positive HPV tests but negative Pap smears. PLoS One 10: e0140336, 2015.

37 Jung A, Schrauder M, Oswald U, Knoll C, Sellberg P, Palmqvist $\mathrm{R}$, Niedobitek G, Brabletz T and Kirchner T: The invasion front of human colorectal adenocarcinomas shows co-localization of nuclear beta-catenin, cyclin D1, and p16 ${ }^{\mathrm{INK} 4 \mathrm{~A}}$ and is a region of low proliferation. Am J Pathol 159: 1613-1617, 2001.

38 Natarajan E, Saeb M, Crum CP, Woo SB, McKee PH and Rheinwald JG: Co-expression of p16(INK4A) and laminin 5 gamma2 by microinvasive and superficial squamous cell carcinomas in vivo and by migrating wound and senescent keratinocytes in culture. Am J Pathol 163: 477-491, 2003.

39 Palmqvist R, Rutegard JN, Bozoky B, Landberg G and Stenling $\mathrm{R}$ : Human colorectal cancers with an intact p16/cyclin D1/pRb pathway have up-regulated p16 expression and decreased proliferation in small invasive tumor clusters. Am J Pathol 157: 1947-1953, 2000.

40 Svensson S, Nilsson K, Ringberg A and Landberg G: Invade or proliferate? Two contrasting events in malignant behavior governed by p16(INK4a) and an intact Rb pathway illustrated by a model system of basal cell carcinoma. Cancer Res 63: 1737-1742, 2003.

41 Witkiewicz AK, Rivadeneira DB, Ertel A, Kline J, Hyslop T, Schwartz GF, Fortina $P$ and Knudsen ES: Association of $\mathrm{RB} / \mathrm{p} 16$-pathway perturbations with DCIS recurrence: Dependence on tumor versus tissue microenvironment. Am J Pathol 179: 1171-1178, 2011.

42 Schneider-Stock R, Boltze C, Lasota J, Peters B, Corless CL, Ruemmele P, Terracciano L, Pross M, Insabato L, Di Vizio D, Iesalnieks I, Dirnhofer S, Hartmann A, Heinrich M, Miettinen M, Roessner A and Tornillo L: Loss of p16 protein defines highrisk patients with gastrointestinal stromal tumors: a tissue microarray study. Clin Cancer Res 11: 638-645, 2005.

43 Ayhan S, Isisag A, Saruc M, Nese N, Demir MA and Kucukmetin NT: The role of pRB, p16 and cyclin D1 in colonic carcinogenesis. Hepatogastroenterology 57: 251-256, 2010.

Received April 4, 2018

Revised May 10, 2018

Accepted May 15, 2018 